\title{
Modeling the Transport of Hydrocarbons in the Subsurface Environment with Chemical Reaction
}

\author{
S. V. Hemalatha, Nirmala P. Ratchagar
}

\begin{abstract}
The objective of the present study is to investigate the transport of hydrocarbons with chemical reaction due to oil flow through the subsurface. The coupled nonlinear differential equations governing the flow and mass transfer are simplified using perturbation technique and solved numerically. The dimensionless velocity and concentration profiles are depicted graphically and discussed for the effects of the parameters involved.
\end{abstract}

Keywords: Beavers-Joseph slip condition, Buoyancy effects, Concentration of hydrocarbons, Retardation.

\section{INTRODUCTION}

Solute transport problems involving first order decay reactions and retardation coefficient frequently occurs in soil and groundwater systems. Thus the migration of petroleum hydrocarbon products through the soil causes physical, chemical and biochemical changes depending not only on their own properties, but also on soil properties[1].

Some of the petrochemical products get adsorbed on soil when entering the subsurface and reach the underground water table[2, 3]. Experimental result of Adam et al.[4] showed that addition of oxygenates such as ethanol, reduces the atmospheric pollution, but increase the downward migration of hydrocarbons to groundwater by lessening the adsorptive capacity of the surface soil components. Transport of polycyclic aromatic hydrocarbons in porous medium is studied by Sabbah et al. [5] using break through curves. Mukherjee and Shome [6] presented an analytical solution for a double phase flow of water and oil in saturated porous medium using calculus of variation and similarity theory.

Gawdzik and Zygadlo [7] experimentally verified the results of contamination by hydrocarbons migration in the soil using soil filter test. Abdulkadir and Yahaya[8] studied the dispersion of crude oil on land with the help of MINITAB software. Okop and Ekpo [9] classified the hydrocarbon penetration due to crude oil spillage in the soil depths according to their chemical similarities.

Our objective is to study the movement and characteristics of oil flow during the transport of hydrocarbons in the soil. Soil

Revised Manuscript Received on December 15, 2019.

* Correspondence Author

S. V. Hemalatha*, Department of Mathematics, Kalasalingam Academy of Research and Education, Krishnankoil, India. Email: hemalathasvam@gmail.com

Nirmala P. Ratchagar, Department of Mathematics, Annamalai University, Annamalainagar, India. Email: nirmalapasala@yahoo.co.in in the subsurface under study is bounded by porous layers on both the upper and lower surfaces. The soil is assumed to be a homogeneous porous medium. The fluids are considered to be incompressible. The transient, two dimensional flow of oil, diffuses and simultaneously undergoes first-order irreversible chemical reaction in the liquid under isothermal conditions. The obtained governing equations are solved using perturbation method.

\section{MATHEMATICAL FORMULATION}

The mathematical model is a nonlinear system of equations, based on the principle of mass, linear momentum in the presence of concentration buoyancy effects including Darcian term and the solute transport equation with chemical reaction. These results in following equations as:

$\frac{\partial u}{\partial x}+\frac{\partial v}{\partial y}=0$

$\frac{\partial u}{\partial t}+u \frac{\partial u}{\partial x}+v \frac{\partial u}{\partial y}=v\left(\frac{\partial^{2} u}{\partial x^{2}}+\frac{\partial^{2} u}{\partial y^{2}}\right)+g \beta_{C}\left(C-C_{1}\right)-\frac{v}{k_{p}} u$

$\frac{\partial v}{\partial t}+u \frac{\partial v}{\partial x}+v \frac{\partial v}{\partial y}=v\left(\frac{\partial^{2} v}{\partial x^{2}}+\frac{\partial^{2} v}{\partial y^{2}}\right)-\frac{v}{k_{p}} v$

$\rho_{b} \frac{\partial S}{\partial t}+\beta_{w} \frac{\partial C}{\partial t}+\beta_{w} u \frac{\partial C}{\partial x}+\beta_{w} v \frac{\partial C}{\partial y}=\beta_{w} D\left(\frac{\partial^{2} C}{\partial x^{2}}+\frac{\partial^{2} C}{\partial y^{2}}\right)$

$$
-\beta_{w} k_{1}\left(C-C_{1}\right)
$$

where, $u$ and $v$ are the components of velocities along $x$ and $y$ directions, respectively, $C$ is the concentration of hydrocarbons in water, $S$ is the concentration of adsorbed oil in soil, $v$ is the kinematic viscosity of oil, $g$ is the gravitational acceleration, $\beta_{c}$ is the concentration expansion coefficient, $C_{1}$ is the concentration at the upper surface, $\rho$ is the density of oil, $C_{p}$ is the specific heat at constant pressure, $k_{p}$ is the permeability of the medium, $\rho_{b}$ is the soil bulk density, $\beta_{w}$ is the volumetric water content of soil,

$D$ is the mass diffusivity and $k_{1}$ is the chemical reaction rate parameter.

Accounting for equilibrium in linear sorption process,

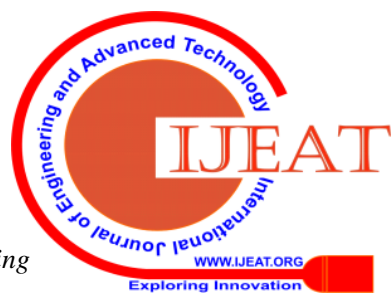


the retardation factor $R=1+\frac{\rho_{b} k_{d}}{\beta_{w}} \quad$ where, $S=k_{d} C, k_{d}$ is the adsorption coefficient, reduces equation (4) to

$R \frac{\partial C}{\partial t}+u \frac{\partial C}{\partial x}+v \frac{\partial C}{\partial y}=D\left(\frac{\partial^{2} C}{\partial x^{2}}+\frac{\partial^{2} C}{\partial y^{2}}\right)-k_{1}\left(C-C_{1}\right)$

Using the appropriate boundary conditions such as the Beavers-Joseph slip condition [10] for horizontal velocity, we have:

$\left.\begin{array}{l}\frac{\partial u}{\partial y}=-\frac{\alpha}{\sqrt{k_{p}}}(u-Q), v=0, C=C_{0}+\varepsilon e^{n t+\lambda x}\left(C_{0}-C_{1}\right) \text { at } y=0 \\ \frac{\partial u}{\partial y}=\frac{\alpha}{\sqrt{k_{p}}}(u-Q), v=-\varepsilon e^{n t+\lambda x} u_{0}, C=C_{1} \text { at } y=h\end{array}\right\}$

where, $\alpha$ is the slip parameter and $C_{0}$ are the temperature and concentration at the lower surface, respectively, $u_{0}, n$ and $\lambda$ are constants and $\varepsilon$ is the perturbation parameter $(<1)$.

We now introduce the following non-dimensional quantities:

$u^{*}=\frac{u}{u_{0}}, v^{*}=\frac{v}{u_{0}}, t^{*}=\frac{t u_{0}^{2}}{v}, x^{*}=\frac{x u_{0}}{v}, y^{*}=\frac{y u_{0}}{v}$,

$\phi=\frac{C-C_{1}}{C_{0}-C_{1}}, Q^{*}=\frac{Q}{u_{0}}$

where, $\phi$ is the dimensionless concentration.

Making use of the non-dimensional variables in equations (1) to (3) and (5) neglecting the '*' symbol gives

$\frac{\partial u}{\partial x}+\frac{\partial v}{\partial y}=0$

$\frac{\partial u}{\partial t}+u \frac{\partial u}{\partial x}+v \frac{\partial u}{\partial y}=\frac{\partial^{2} u}{\partial x^{2}}+\frac{\partial^{2} u}{\partial y^{2}}+G c \phi-\sigma^{2} u$

$\frac{\partial v}{\partial t}+u \frac{\partial v}{\partial x}+v \frac{\partial v}{\partial y}=\frac{\partial^{2} v}{\partial x^{2}}+\frac{\partial^{2} v}{\partial y^{2}}-\sigma^{2} v$

$R \frac{\partial \phi}{\partial t}+u \frac{\partial \phi}{\partial x}+v \frac{\partial \phi}{\partial y}=\frac{1}{S c}\left(\frac{\partial^{2} \phi}{\partial x^{2}}+\frac{\partial^{2} \phi}{\partial y^{2}}\right)-K \phi$

where,

$G c=\frac{v g \beta_{C}\left(C_{0}-C_{1}\right)}{u_{0}{ }^{3}}$ is the mass Grashof number,

$\sigma=\frac{v}{u_{0} \sqrt{k_{p}}}$ is the porous parameter,

$S c=\frac{v}{D}$ is the Schmidt number and
$K=\frac{k_{1} v}{u_{0}{ }^{2}}$ is the dimensionless chemical reaction rate

parameter.

The boundary conditions (6) in non-dimensional form are:

$\left.\begin{array}{l}\frac{\partial u}{\partial y}=-\alpha \sigma(u-Q), v=0, \phi=1+\varepsilon e^{n t+\lambda x} \text { at } y=0 \\ \frac{\partial u}{\partial y}=\alpha \sigma(u-Q), v=-\varepsilon e^{n t+\lambda x}, \phi=0 \text { at } y=1\end{array}\right\}$

\section{METHOD OF SOLUTION}

Applying the perturbation method in the form,

$$
\left.\begin{array}{l}
u(x, y, t)=u_{0}(y)+\varepsilon e^{n t+\lambda x} u_{1}(y)+O\left(\varepsilon^{2}\right) \\
v(x, y, t)=\varepsilon e^{n t+\lambda x} v_{1}(y)+O\left(\varepsilon^{2}\right) \\
\phi(x, y, t)=\phi_{0}(y)+\varepsilon e^{n t+\lambda x} \phi_{1}(y)+O\left(\varepsilon^{2}\right)
\end{array}\right\}
$$

the system of partial differential equations (8) to (10), reduces to a system of ordinary differential equations. Neglecting the higher order of $\left(\varepsilon^{2}\right)$ gives the following set of equations for $u_{0}, \phi_{0}$ and $u_{1}, v_{1}, \phi_{1}$ in simplified form.

Zeroth order equations:

$u_{0 y y}-\sigma^{2} u_{0}+G c \phi_{0}=0$

$\phi_{0 y y}-S c K \phi_{0}=0$

First order equations:

$u_{1 y y}+\left(\lambda^{2}-\sigma^{2}-\lambda u_{0}-n\right) u_{1}-v_{1} u_{0 y}+G c \phi_{1}=0$

$v_{1 y y}+\left(\lambda^{2}-\sigma^{2}-\lambda u_{0}-n\right) v_{1}=0$

$\left.\phi_{1 y y}+\left(\lambda^{2}-S c\left(\lambda u_{0}+n R+K\right)\right) \phi_{1}-S c v_{1} \phi_{0 y}\right)=0$

subject to the boundary conditions,

$u_{0 y}=-\alpha \sigma\left(u_{0}-Q\right), \quad \phi_{0}=1$ at $y=0$

$u_{0 y}=\alpha \sigma\left(u_{0}-Q\right), \quad \phi_{0}=0$ at $\left.y=1\right\}$

$u_{1 y}=-\alpha \sigma u_{1}, v_{1}=0, \phi_{1}=0$ at $y=0$

$u_{1 y}=\alpha \sigma u_{1}, v_{1}=-1, \phi_{1}=0$ at $\left.y=1\right\}$

The numerical solutions for the coupled equations (13)-(17) using the boundary conditions (18) and (19) give the velocity profiles and concentration distributions.

\section{RESULTS AND DISCUSSION}

Numerical evaluation for the velocity profiles and concentration distributions have been computed using MATHEMATICA 8.0. To understand the nature of movement of oil spill and the distribution of concentration of hydrocarbons in the real field, physical numbers for Schmidt number, chemical reaction rate parameter, retardation factor and porous parameter are chosen and the results obtained 
are discussed through Figures 1 to 6 .

The values of other physical parameters are set as real constants.

Figures 1 and 2 indicate the velocity(u) and the velocity(v) for different porous parameter, respectively. From Figure 1, it is seen that the velocity decreases with increase in porous parameter. This is due to the frictional drag resistance against the flow. But for vertical flow, the velocity(v) increases for increasing porous parameter as in Figure 2.

Figures 3 and 4 show the effect of Schmidt number and chemical reaction rate parameter on the concentration profile, respectively. It reveals that the concentration decreases with increasing Schmidt number and chemical reaction rate parameter. This causes the concentration buoyancy to decrease yielding a reduction in the fluid velocity.

Figures 5 and 6 signify the porous parameter and retardation factor of concentration profile with respect to time. They indicate that concentration of hydrocarbon in water increases with increase in time. Figure 5 shows that the concentration increases with increasing porous parameter. From Figure 6, we see that when the retardation factor increases the concentration decreases. This shows less retardation factor at higher concentrations, and higher retardation factor at lower concentrations.

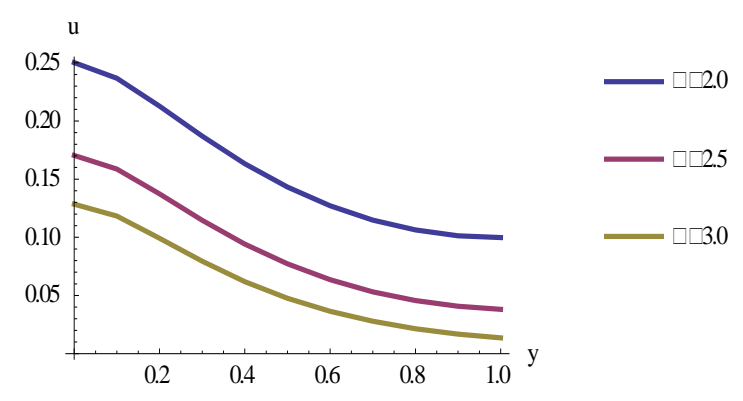

Fig. 1. Effect of porous parameter on u velocity

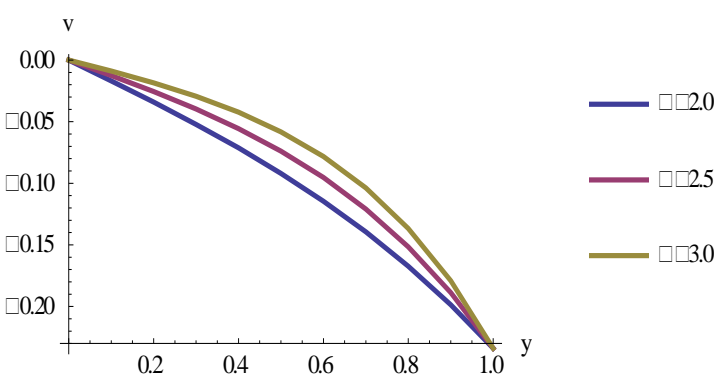

Fig. 2. Effect of porous parameter on $v$ velocity

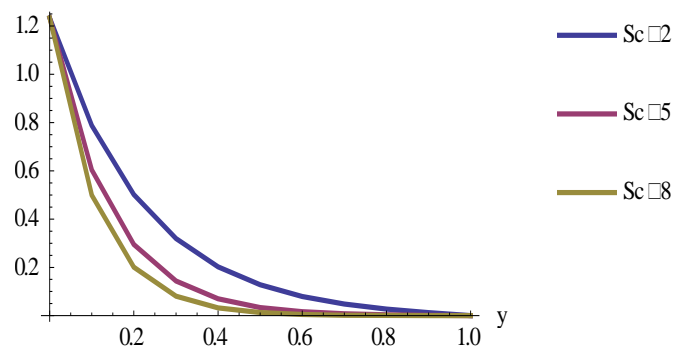

Fig. 3. Effect of Schmidt number on concentration distribution

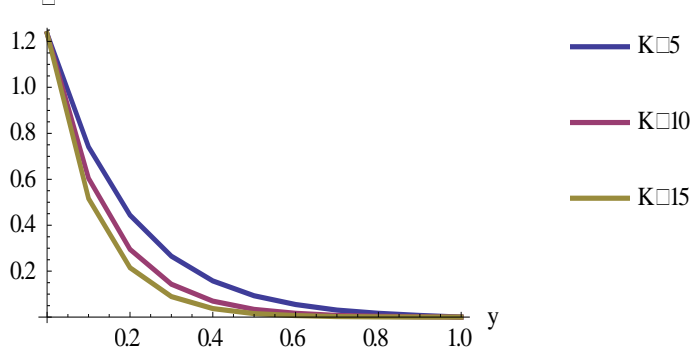

Fig. 4. Effect of chemical reaction rate parameter on concentration distribution

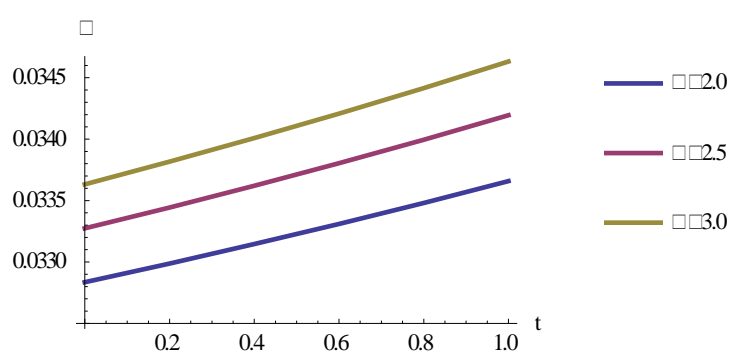

Fig. 5. Time evolution of concentration distribution for different porous parameter

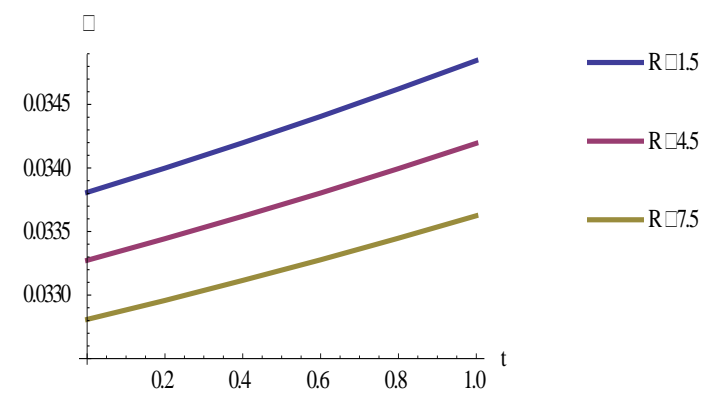

Fig. 6. Time evolution of concentration distribution for different retardation parameter

\section{CONCLUSION}

Transport problems are in much need of multi-dimensional solutions as they widely applied to solute dispersal in any direction. This paper is focused on the study of transport of hydrocarbons through the subsurface including chemical reaction. The numerical solution predicts the evolution of oil penetration into soil and its characteristics that describe the transport of hydrocarbons dissolved in the water, both in the period immediately after the spill, and over the subsequent time. Obtained results may serve as a predictive tool in environmental / groundwater management and applies to real-world applications.

\section{REFERENCES}

1. Nirmala P Ratchagar and S.V.Hemalatha, Transport of petroleum hydrocarbons in the subsurface with internal heat generation, Malaya Journal of Matematik, S(2), 2015, pp. 496-503

2. M. Abdulkadir and S. Yahaya, Modeling and simulation of the effects of crude oil dispersion on land, ARPN journal of Engineering and Applied Sciences, 6(8), 2011, pp. 26-30. 
3. M. Brown1, Matthijs Bonte, Richard Gill, James Dawick \& Peter J. Boogaard, Heavy hydrocarbon fate and transport in the environment David,

Quarterly Journal of Engineering Geology and Hydrogeology, 50(3), 2017, pp. 142-162.

4. M. Zygadlo and J. Gawdzik, Modeling the Transport of Petroleum Products by Soil Filter Method, Polish Journal of Environmental Studies, 19(4), 2010, pp. 841-847.

5. G. Adam, K. Gamoh, D.G. Morris, and H. Duncan, Effect of alcohol addition on the movement of petroleum hydrocarbon fuels in soil, The Science of the Total Environment, 286, 2002, pp. 15-25.

6. I. Sabbah, M. Rebhun and Z. Gerstl, An independent prediction of the effect of dissolved organic matter on the transport of polycyclic aromatic hydrocarbons, Journal of Contaminant Hydrology, 75(1-2), 2004, pp. 55-70.

7. B. Mukherjee, B and P. Shome, An analytic solution of fingering phenomenon arising in fluid flow through porous media by using techniques of calculus of variation and similarity theory\}, Journal of Mathematics Research, 1(2), 2009, pp. 64-72.

8. J.Gawdzik and M. Zygadlo, Modelling transport of hydrocarbons in soil-water environment, Ecological Chemistry and Engineering Science, 17(3), 2010, pp. 331-343.

9. I. J. Okop and S. C. Ekpo, Determination of total hydrocarbon content in soil after petroleum spillage, Proceedings of the World Congress on Engineering, London, U.K., 3, 2012.

10. G.S.Beavers and D.D. Joseph, Boundary condition at a naturally permeable wall, Journal of Fluid Mechanics, 30, 1967, pp. 197-207.

\section{AUTHORS PROFILE}

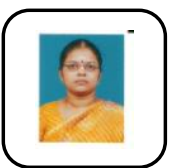

Dr. S. V. Hemalatha, is currently working as an Assistant Professor in the Department of Mathematics, Kalasalingam Academy of Research and Education, India. She obtained her Ph.D. Mathematics in 2017 from Annamalai University, India. She has published 7 research papers in various International Journals and Conferences. Her area of interest is Fluid Dynamics.

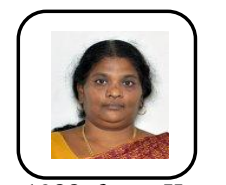

Dr. Nirmala P. Ratchagar, is currently working as a Professor(Former Head) in the Department of Mathematics, Annamalai University, India. She completed her M.Sc. Mathematics in 1985 from IIT, Kanpur, India and obtained her Ph.D. Mathematics in 1988 from Kanpur University, India. She has published more than 45 research papers in various International Journals and Conferences. Her area of interest includes Fluid Dynamics - Bio- Mechanics, Fluid transport process, optimization and epidemics. 